Supporting Information for

\title{
Decomposition of the Simulant 2-Chloroethyl Ethyl Sulfide Blister Agent under Ambient Conditions Using Metal Organic Frameworks
}

Hong-Hyun Kim ${ }^{\mathrm{a}}$, Jin Young Seo ${ }^{\mathrm{b}}$, Heejeong Kimc, Sangjo Jeonga ${ }^{\mathrm{a}}$, Kyung-Youl Baek ${ }^{\mathrm{b}}$, Jongsik

$\mathrm{Kim}^{\mathrm{c}}$, Sein Minc , Sang Hoon Kim${ }^{\mathrm{b}}$, Keunhong Jeong ${ }^{\mathrm{c} *}$

a.Department of Civil Engineering and Environmental Engineering Science and Nuclear \& WMD Protection Research Center, Korea Military Academy, Seoul, 01805, Republic of Korea.

b.Materials Architecturing Research Center, Korea Institute of Science and Technology, Hwarangno 14-gil 5, Seongbuk-gu, Seoul, 02792, Republic of Korea.

c.Department of Chemistry and Nuclear \& WMD Protection Research Center, Korea Military Academy, Seoul, 01805, Republic of Korea.

Corresponding authors: S. H. Kim (Kim_sh@kist.re.kr), K. Jeong (doas1mind@kma.ac.kr)

$\dagger$ Contributed equally to this paper 


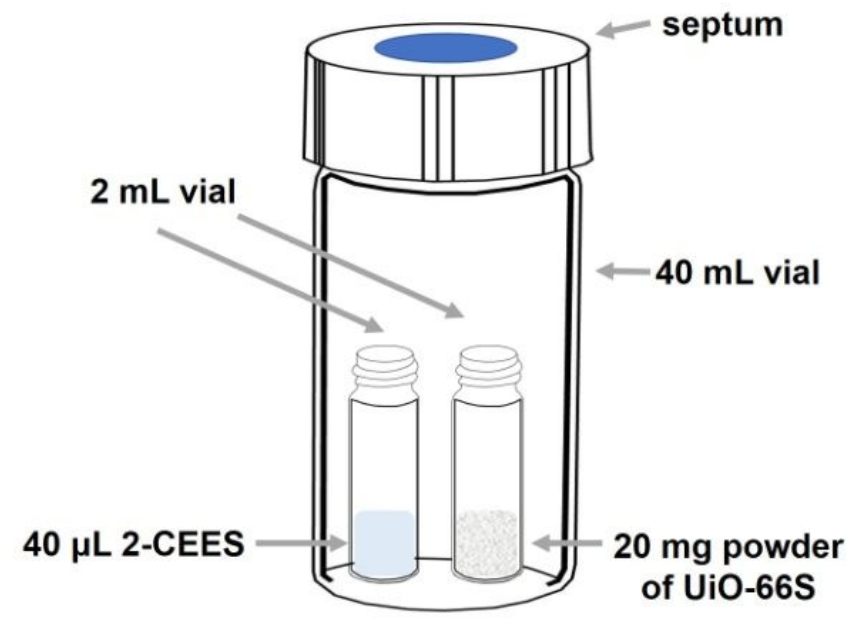

Figure S1. Schematic illustration of Vials in vial cloased gas phase decomposition system.

\section{Vials-in-Vial closed gas phase decomposition system (ViV)}

The decomposition of the 2-CEES vapors were investigated in batch experiments using a Vials-in-Vial closed gas phase decomposition system. A $1 \mathrm{~mL}$ glass vial containing $20 \mathrm{mg}$ of the UiO-66S catalyst was placed into a $40 \mathrm{~mL}$ reaction vessel. The liquid form of the 2-CEES (40 $\mu$ L) was injected using a syringe to a second vial that was placed inside the reaction vessel. The later was immediately hermetically sealed using a cap with a septum. A schematic illustration of the $\mathrm{ViV}$ is presented above. The vessels were kept at a room temperature. After $24 \mathrm{~h}$ reaction, the n-heptane $(1 \mathrm{~mL})$ was introduced into the UiO-66S vessel to identify the species produced. The sample was also analyzed by GC-MS. 
$24 \mathrm{~h}$

$18 \mathrm{~h}$

$12 \mathrm{~h}$

$6 \mathrm{~h}$

Oh

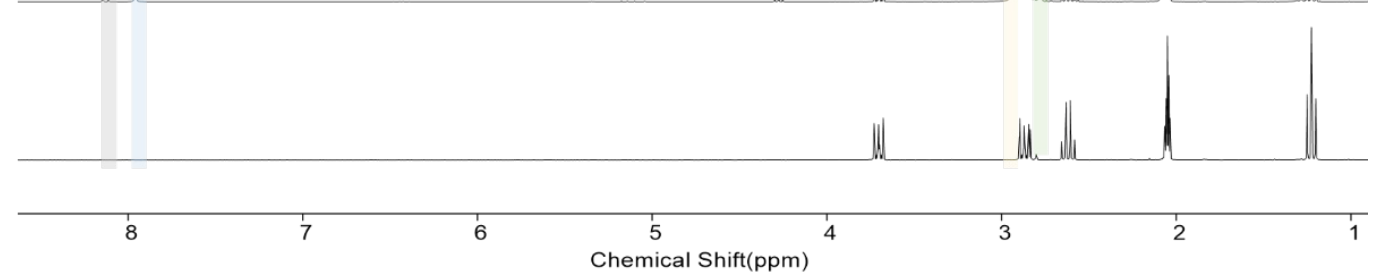

Figure S2. ${ }^{1} \mathrm{H}-\mathrm{NMR}$ spectra with different 2-CEES decomposition time using UiO-66S as catalyst

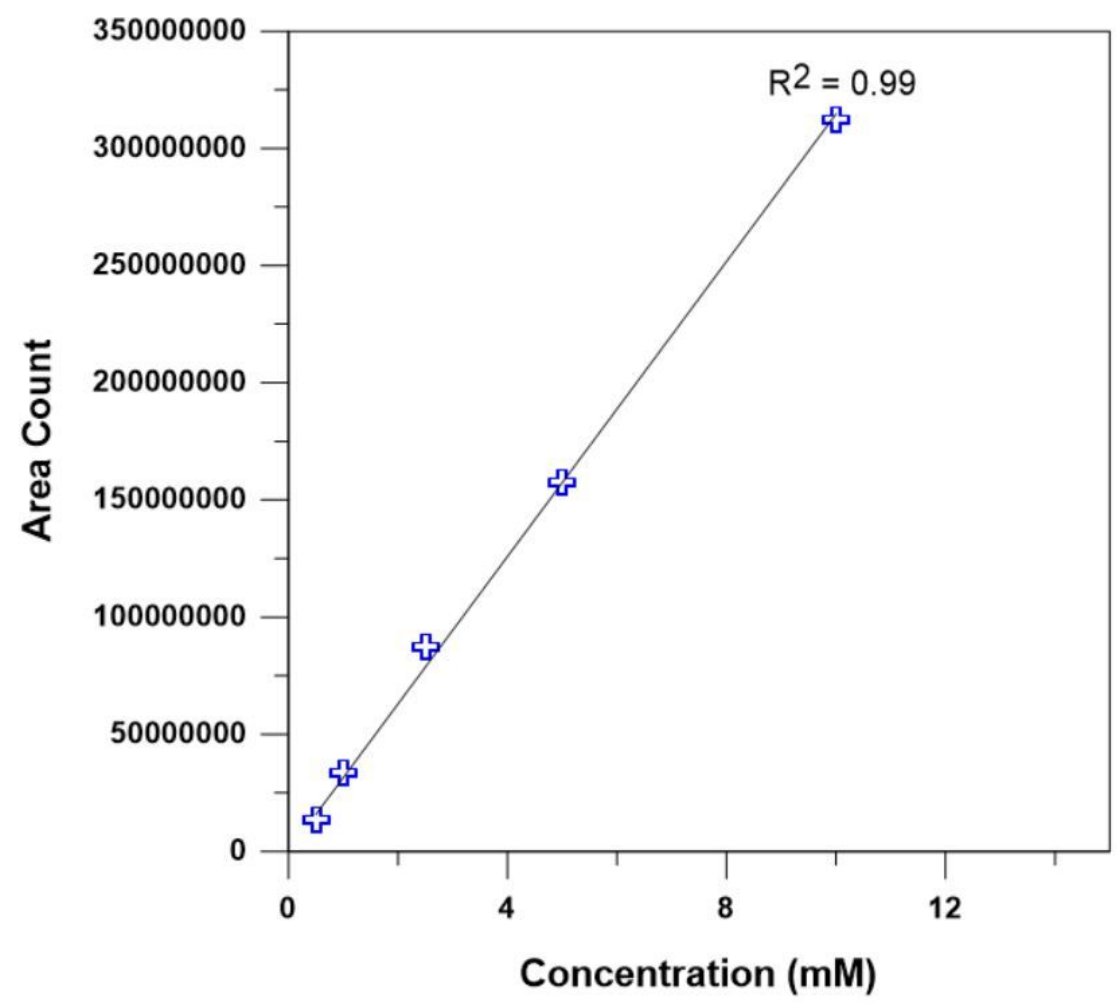

Figure S3. correlation between concentration of THT and integrated GC area 


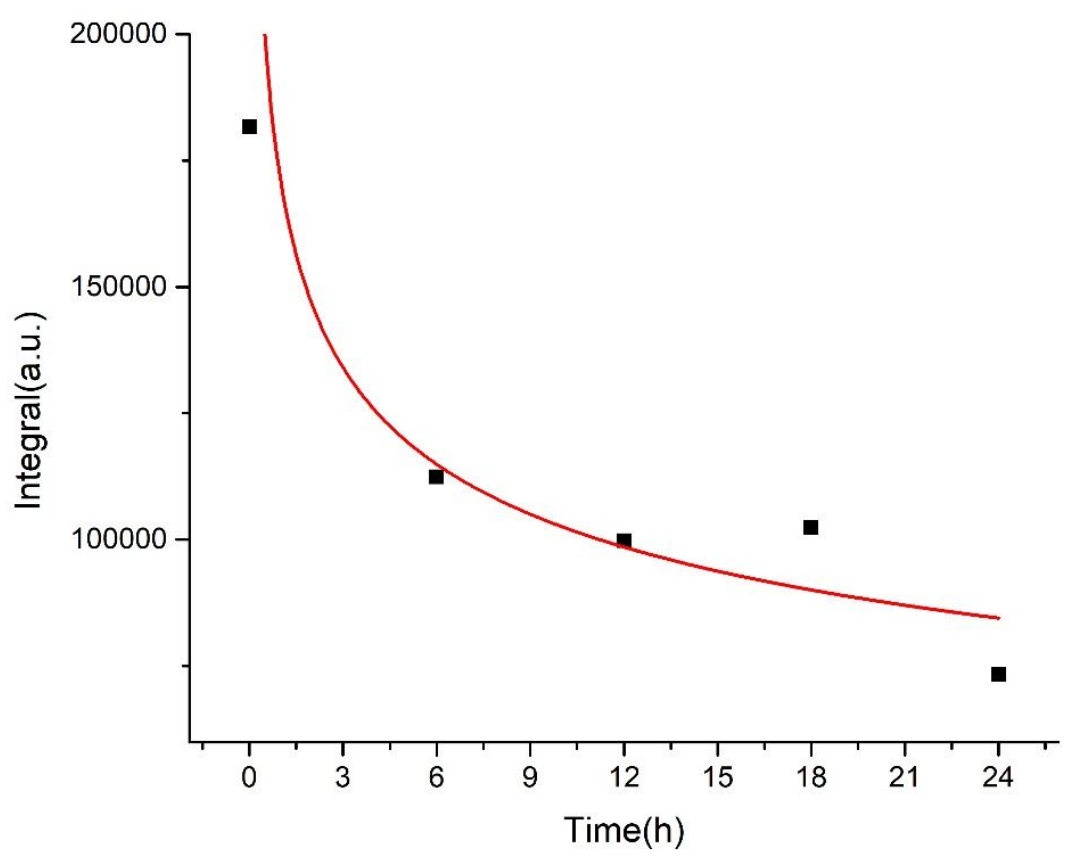

Figure S4. Integrated NMR signal around $1.2 \mathrm{ppm}$ along the reaction time (2-CEES)

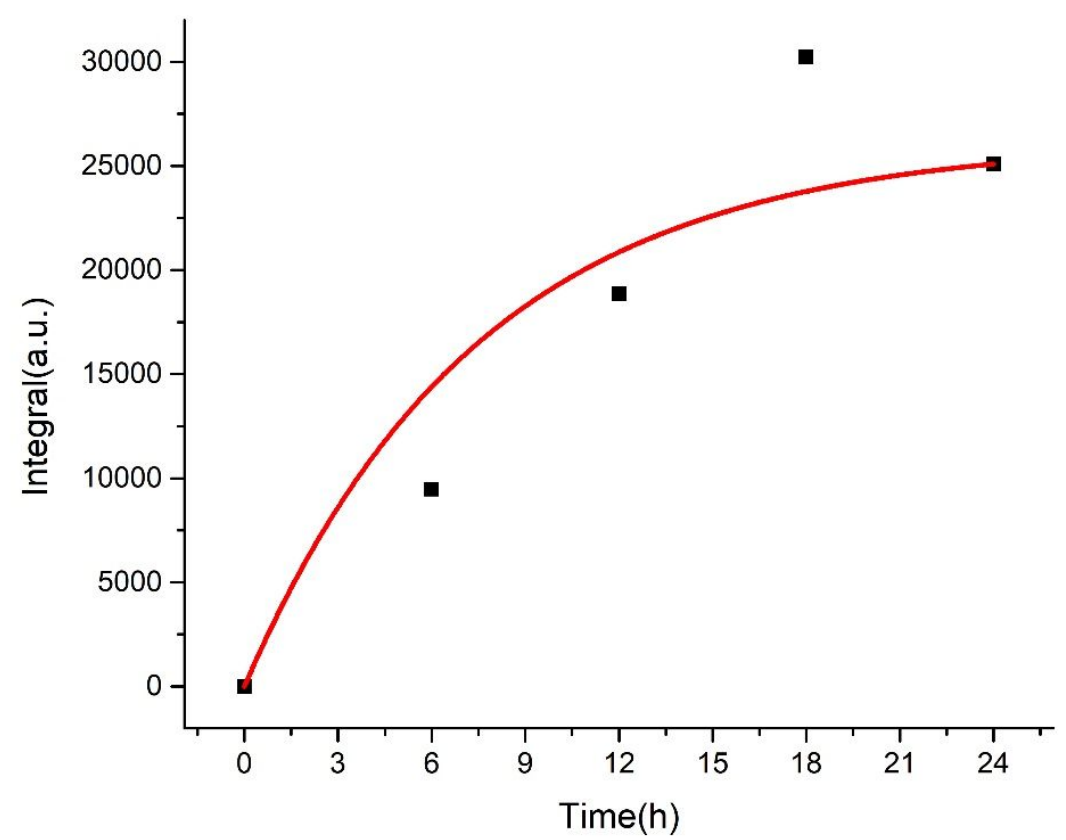

Figure S5. Integrated NMR signal around $8.2 \mathrm{ppm}$ along the reaction time (THT) 


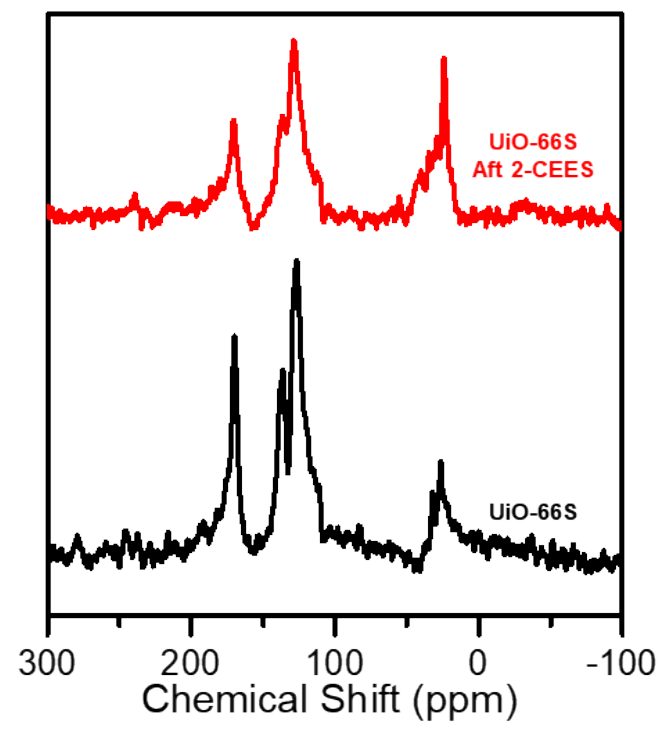

Figure S6. Solid state ${ }^{13} \mathrm{C}$ NMR spectra of UiO-66S, and UiO-66S after 2-CEES decomposition

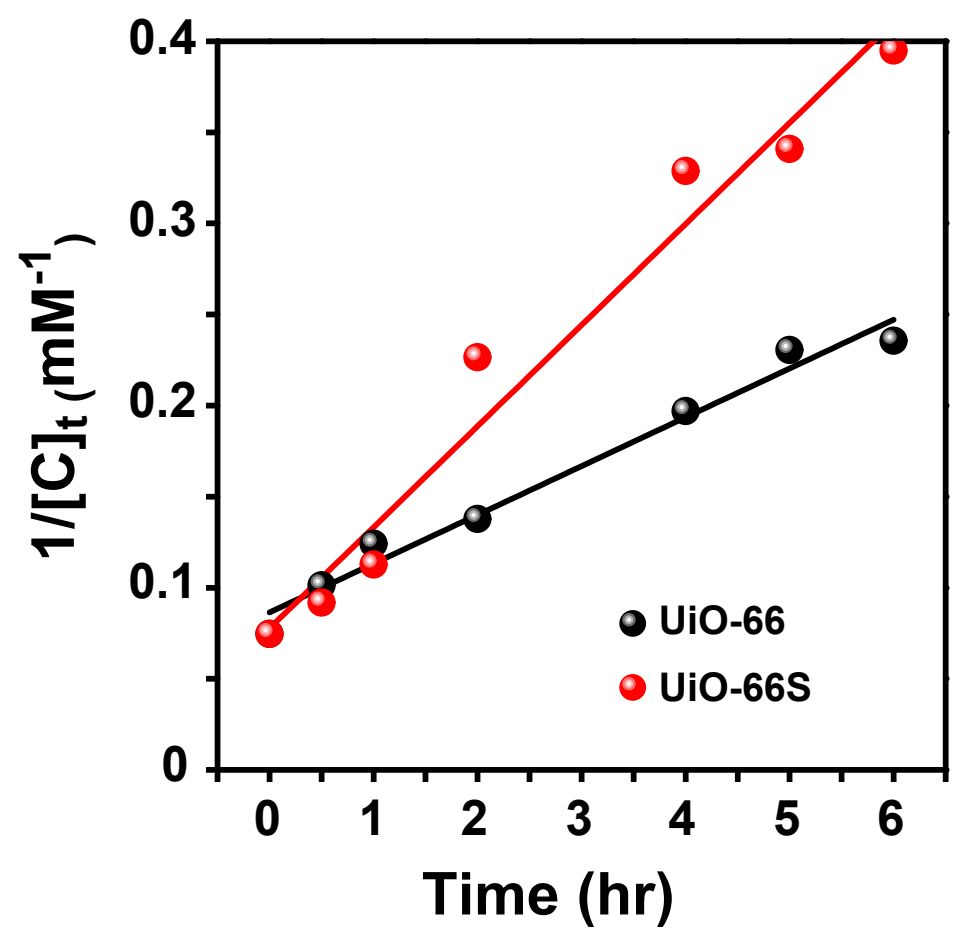

Figure S7. Linear plot of $1 /[\mathrm{C}]_{\mathrm{t}}$ vs time for 2-CEES decomposition using UiO-66 and UiO-66S as catalyst. 


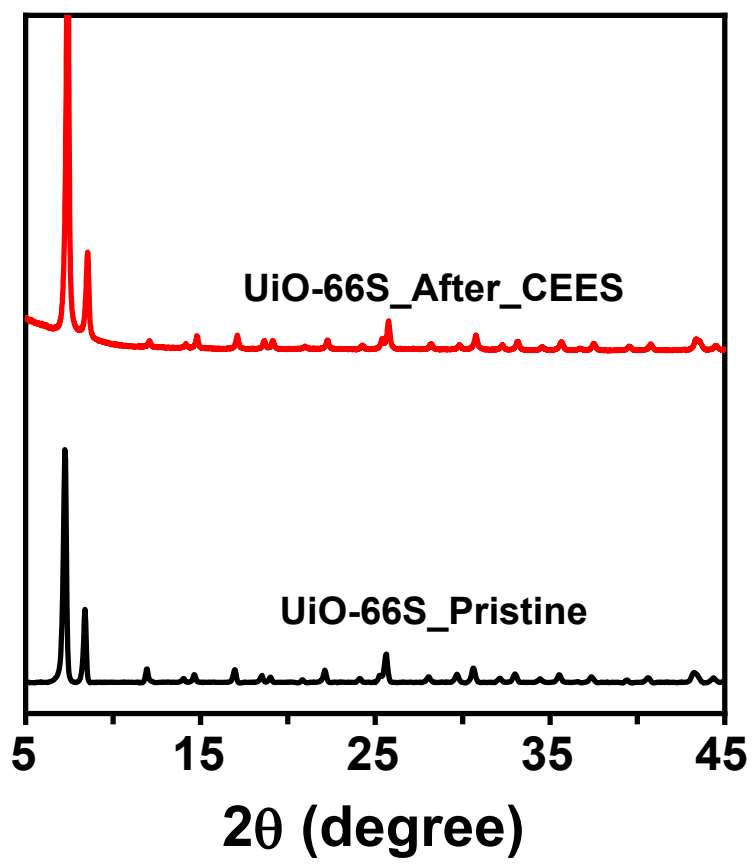

Figure S8. XRD patterns of pristineUiO-66S, and UiO-66S after 2-CEES degradation.

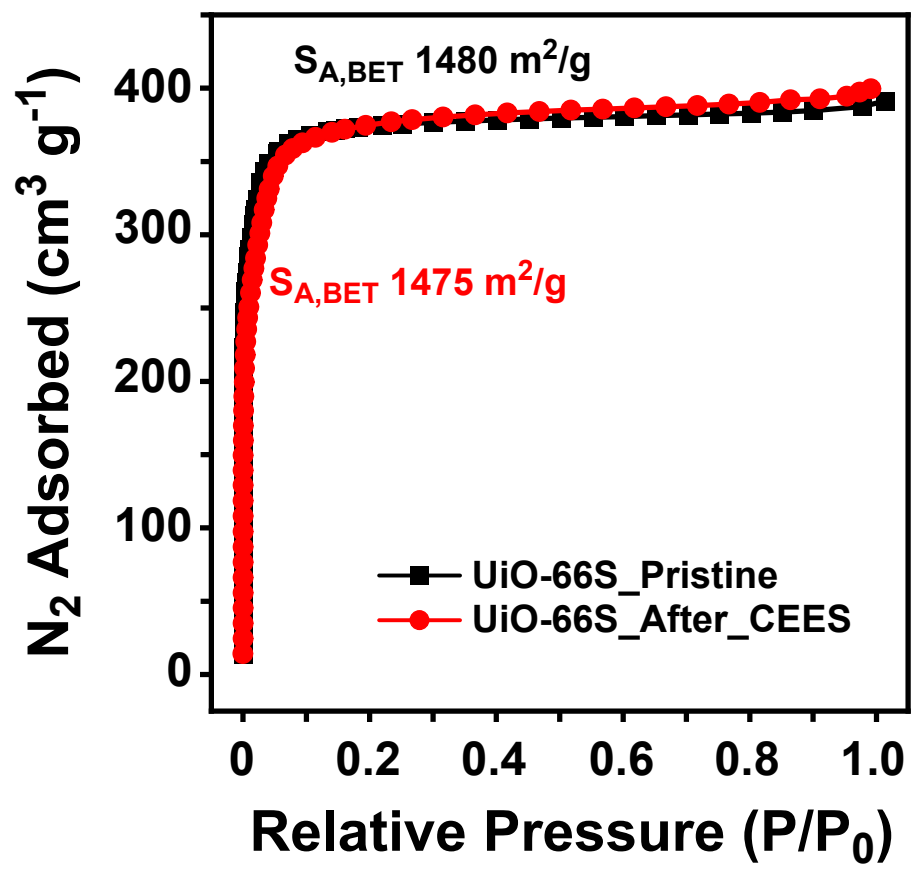

Figure S9. $\mathrm{N}_{2}$ adsorption isotherm at $77 \mathrm{~K}$ of UiO-66S, and UiO-66S after 2-CEES degradation. 


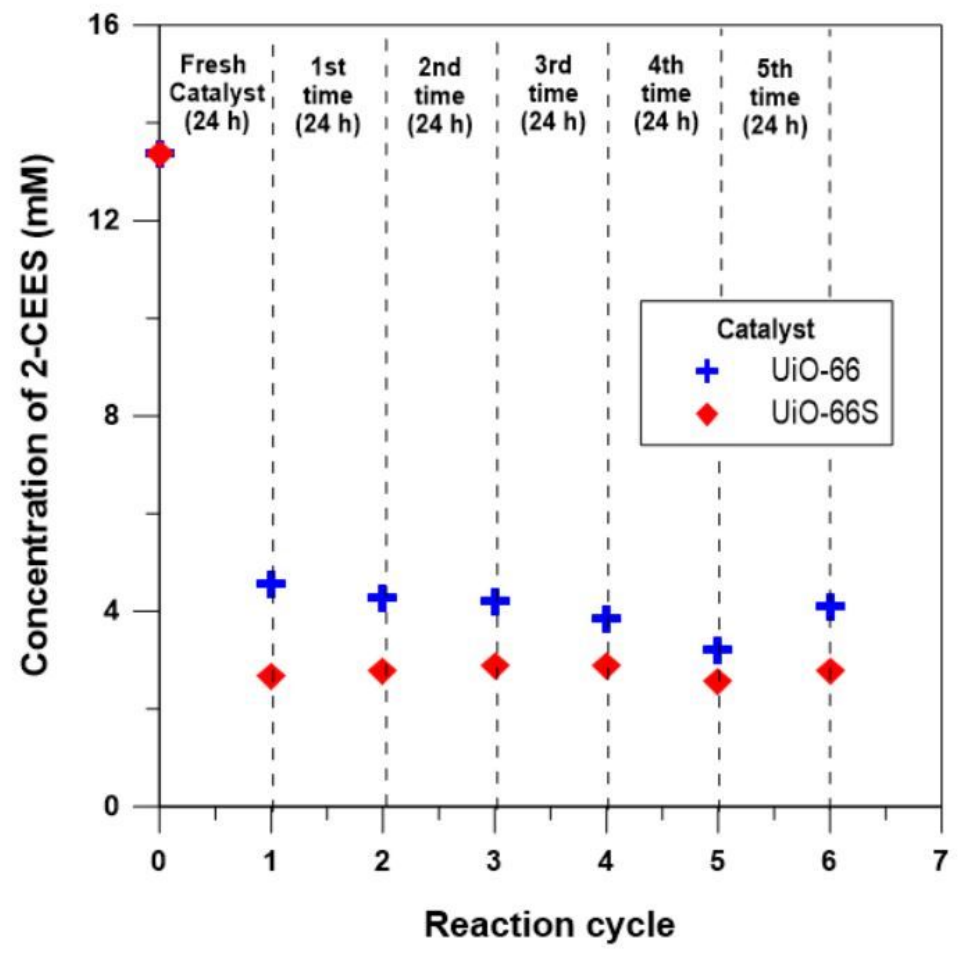

Figure S10. Results of 2-CEES decomposition reaction for 24 hours after each reusability test for two catalysts (UiO-66 and UiO-66S) 


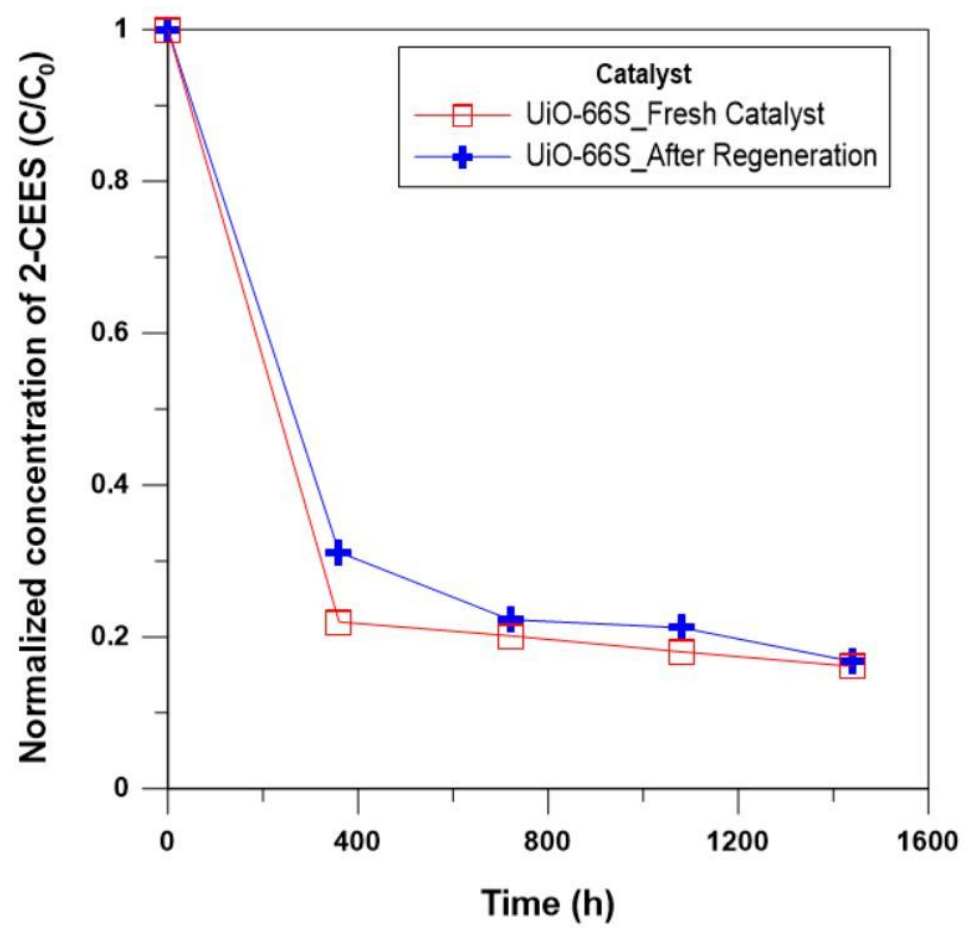

Figure S11. Decomposition performance tests using fresh catalyst and regenerated catalyst Table S1. GC-MS analysis table for 2-CEES destruction at $24 \mathrm{hr}$ using UiO-66S as catalysts.

\begin{tabular}{cccccc}
\hline Entry & $\begin{array}{c}\text { Retention Time } \\
(\mathbf{m i n})\end{array}$ & Compound Name & $\begin{array}{c}\text { Molecular } \\
\text { Formula }\end{array}$ & $\begin{array}{c}\text { Molecular } \\
\text { Weight } \\
(\mathbf{g} / \mathbf{m o l})\end{array}$ & $\begin{array}{c}\text { Peak } \\
\text { Area } \\
(\%)\end{array}$ \\
\hline 1 & 4.72 & 2-hydroxyethylethylsulfide & $\mathrm{C}_{4} \mathrm{H}_{10} \mathrm{OS}$ & 106.19 & 2.79 \\
\hline 2 & 4.83 & 2-CEES & $\mathrm{C}_{4} \mathrm{H}_{9} \mathrm{ClS}$ & 124.63 & 68.86 \\
\hline 3 & 5.87 & Tetrahydrothiophene & $\mathrm{C}_{4} \mathrm{H}_{8} \mathrm{~S}$ & 88.1 & \multirow{2}{*}{25.72} \\
\cline { 2 - 4 } & & Thiophene & $\mathrm{C}_{4} \mathrm{H}_{4} \mathrm{~S}$ & 84.1 & \\
\hline
\end{tabular}


Coordiates of studied structures of DFT

TiO-66S surface and 2-CEES

$\begin{array}{lccc}\mathrm{C} & 4.53821700 & 3.13746800 & 2.98758300 \\ \mathrm{C} & -4.53784600 & 2.98690600 & -3.13813700 \\ \mathrm{C} & 4.54403400 & 3.00311100 & -3.11946700 \\ \mathrm{C} & -4.54361300 & 3.12012300 & 3.00253800 \\ \mathrm{C} & -4.54338000 & -3.11995500 & -3.00310800 \\ \mathrm{C} & 4.54358200 & -3.00260900 & 3.12053400 \\ \mathrm{C} & -4.53824000 & -2.98754400 & 3.13700200 \\ \mathrm{C} & 4.53842900 & -3.13763200 & -2.98699300 \\ \mathrm{Zr} & -2.51940600 & 0.00002500 & -0.00015100 \\ \mathrm{Zr} & 2.51970900 & -0.00001100 & 0.00014900 \\ \mathrm{Zr} & 0.00048800 & 1.68419600 & 1.66051100 \\ \mathrm{Zr} & -0.00018000 & 1.66051600 & -1.68418200 \\ \mathrm{Zr} & -0.00031600 & -1.66049500 & 1.68419100 \\ \mathrm{Zr} & 0.00061200 & -1.68417600 & -1.66050000 \\ \mathrm{O} & 3.70649700 & -1.49498200 & 1.50868800 \\ \mathrm{O} & 3.70185400 & -1.50999800 & -1.49629700 \\ \mathrm{O} & 2.21954900 & -2.67699600 & -2.72365400 \\ \mathrm{O} & 2.22431200 & -2.73048700 & 2.66648700 \\ \mathrm{O} & -0.00127200 & -1.88202100 & -4.32618900 \\ \mathrm{O} & -0.03978600 & -4.37541600 & -1.53579700 \\ \mathrm{O} & 0.00152500 & -4.32620200 & 1.88199900 \\ \mathrm{O} & 3.70666500 & 1.49491700 & -1.50830900 \\ \mathrm{O} & 3.70178800 & 1.51000100 & 1.49662900 \\ \mathrm{O} & 2.22465900 & 2.73060900 & -2.66611700 \\ \mathrm{O} & 2.21934300 & 2.67704900 & 2.72376300 \\ \mathrm{O} & 1.28294200 & -0.00846800 & -1.82112300 \\ \mathrm{O} & 1.28280600 & 0.00847700 & 1.82133800 \\ \mathrm{O} & -1.28154400 & -0.00853000 & -1.82537000 \\ \mathrm{O} & -3.70624500 & -1.50847500 & -1.49511300 \\ \mathrm{O} & -3.70159300 & -1.49641100 & 1.50981500 \\ \mathrm{O} & -2.22407500 & -2.66632500 & -2.73057700 \\ \mathrm{O} & -2.21930800 & -2.72377900 & 2.67681600 \\ \mathrm{O} & -1.28257400 & -1.82120500 & 0.00841700 \\ \mathrm{O} & -3.70144500 & 1.49653400 & -1.51016800 \\ \mathrm{O} & -3.70629800 & 1.50852600 & 1.49478700 \\ \mathrm{O} & -2.21893600 & 2.72355100 & -2.67717100 \\ \mathrm{O} & -2.22427600 & 2.66630300 & 2.73050300 \\ \mathrm{O} & -1.28256700 & 1.82125600 & -0.00852900 \\ \mathrm{O} & 0.00185700 & 4.32617800 & -1.88207000 \\ \mathrm{O} & -0.04002100 & 4.37542500 & 1.53580400 \\ \mathrm{O} & 1.28191900 & 1.82526800 & -0.00848000\end{array}$




\begin{tabular}{|c|c|c|c|}
\hline $\mathrm{O}$ & 1.28191200 & -1.82527600 & 0.00861300 \\
\hline $\mathrm{O}$ & -1.28168000 & 0.00856300 & 1.82517400 \\
\hline $\mathrm{O}$ & 0.03972000 & -1.53576800 & 4.37545500 \\
\hline $\mathrm{O}$ & -0.00148900 & 1.88202900 & 4.32620500 \\
\hline $\mathrm{H}$ & -0.86223500 & 4.45865400 & 1.03117800 \\
\hline $\mathrm{H}$ & 0.64247100 & 4.47849700 & 0.85650300 \\
\hline $\mathrm{H}$ & -0.75620700 & 4.18596900 & -2.47588000 \\
\hline $\mathrm{H}$ & 0.78390600 & 4.18582300 & -2.44432300 \\
\hline $\mathrm{H}$ & -0.78299900 & 2.44501100 & 4.18582400 \\
\hline $\mathrm{H}$ & 0.75713800 & 2.47507600 & 4.18595300 \\
\hline $\mathrm{H}$ & -0.64286300 & -0.85654500 & 4.47843500 \\
\hline $\mathrm{H}$ & 0.86186300 & -1.03106300 & 4.45888900 \\
\hline $\mathrm{H}$ & 0.78265500 & -4.18581600 & 2.44549900 \\
\hline $\mathrm{H}$ & -0.75750700 & -4.18592800 & 2.47452900 \\
\hline $\mathrm{H}$ & -0.86198000 & -4.45872000 & -1.03115300 \\
\hline $\mathrm{H}$ & 0.64273200 & -4.47847800 & -0.85652100 \\
\hline $\mathrm{H}$ & 0.75731100 & -2.47511900 & -4.18593000 \\
\hline $\mathrm{H}$ & -0.78282300 & -2.44495200 & -4.18581800 \\
\hline $\mathrm{C}$ & 3.39712000 & 2.37268100 & -2.36999100 \\
\hline $\mathrm{C}$ & 3.39190500 & -2.37968000 & -2.36599500 \\
\hline $\mathrm{C}$ & 3.39174400 & 2.37966400 & 2.36631100 \\
\hline $\mathrm{C}$ & 3.39680700 & -2.37252100 & 2.37055500 \\
\hline $\mathrm{C}$ & -3.39137200 & 2.36609200 & -2.38009100 \\
\hline $\mathrm{C}$ & -3.39657600 & -2.37025700 & -2.37274000 \\
\hline $\mathrm{C}$ & -3.39672900 & 2.37032100 & 2.37243700 \\
\hline $\mathrm{C}$ & -3.39166900 & -2.36623000 & 2.37938100 \\
\hline $\mathrm{H}$ & 4.92838900 & -2.27735700 & 3.85211000 \\
\hline $\mathrm{H}$ & 4.24035100 & -3.90460700 & 3.66394900 \\
\hline $\mathrm{H}$ & 5.37472000 & -3.24120600 & 2.44517600 \\
\hline $\mathrm{H}$ & -4.23561900 & -3.88471200 & 3.68869800 \\
\hline $\mathrm{H}$ & -4.92093600 & -2.25472200 & 3.86211800 \\
\hline $\mathrm{H}$ & -5.37072500 & -3.23138700 & 2.46518800 \\
\hline $\mathrm{H}$ & -4.23517100 & 3.88410200 & -3.68989700 \\
\hline $\mathrm{H}$ & -4.91996400 & 2.25392500 & -3.86337100 \\
\hline $\mathrm{H}$ & -5.37062800 & 3.23070300 & -2.46676300 \\
\hline $\mathrm{H}$ & 4.92920500 & 2.27808100 & -3.85106900 \\
\hline $\mathrm{H}$ & 4.24084000 & 3.90518400 & -3.66277900 \\
\hline $\mathrm{H}$ & 5.37490800 & 3.24169100 & -2.44377500 \\
\hline $\mathrm{H}$ & -4.24011500 & -3.66322800 & -3.90518100 \\
\hline $\mathrm{H}$ & -4.92836700 & -3.85162800 & -2.27805100 \\
\hline $\mathrm{H}$ & -5.37439900 & -2.44443600 & -3.24166500 \\
\hline $\mathrm{H}$ & -4.24045500 & 3.66363200 & 3.90450300 \\
\hline $\mathrm{H}$ & -4.92860400 & 3.85158200 & 2.27726700 \\
\hline $\mathrm{H}$ & -5.37460700 & 2.44460700 & 3.24119900 \\
\hline $\mathrm{H}$ & 4.92095900 & 3.86245100 & 2.25465300 \\
\hline $\mathrm{H}$ & 4.23548000 & 3.68932900 & 3.88461100 \\
\hline
\end{tabular}




$\begin{array}{lrrr}\mathrm{H} & 5.37070700 & 2.46574100 & 3.23165100 \\ \mathrm{H} & 4.92060200 & -3.86295500 & -2.25410000 \\ \mathrm{H} & 4.23590900 & -3.68916200 & -3.88429800 \\ \mathrm{H} & 5.37124400 & -2.46610800 & -3.23050100 \\ \mathrm{C} & -2.28886900 & 0.40135900 & -5.00990500 \\ \mathrm{H} & -1.71194500 & -0.46192100 & -5.36553800 \\ \mathrm{H} & -2.42873200 & 0.28484000 & -3.92561100 \\ \mathrm{C} & -1.62004400 & 1.70716600 & -5.36022000 \\ \mathrm{H} & -2.23495300 & 2.57102500 & -5.08105800 \\ \mathrm{H} & -1.41574700 & 1.79285200 & -6.43892200 \\ \mathrm{~S} & -0.01815700 & 2.04302800 & -4.54642300 \\ \mathrm{C} & 1.02309000 & 0.86805800 & -5.48841400 \\ \mathrm{H} & 1.14831500 & 1.28824200 & -6.49749900 \\ \mathrm{H} & 0.45320300 & -0.06586600 & -5.56483400 \\ \mathrm{C} & 2.35993000 & 0.60492500 & -4.84655100 \\ \mathrm{H} & 2.27041700 & 0.24448600 & -3.81172200 \\ \mathrm{H} & 3.00350000 & 1.49150800 & -4.86348500 \\ \mathrm{Cl} & 3.21768800 & -0.67466800 & -5.78016600 \\ \mathrm{H} & -3.27510900 & 0.35094700 & -5.49531800\end{array}$

TiO-66S surface and THT

$\begin{array}{lrrrr}\mathrm{C} & -1 & 4.53821700 & 3.13746800 & 2.98758300 \\ \mathrm{C} & -1 & -4.53779600 & 2.98694900 & -3.13817700 \\ \mathrm{C} & -1 & 4.54403400 & 3.00311100 & -3.11946700 \\ \mathrm{C} & -1 & -4.54361300 & 3.12012300 & 3.00253800 \\ \mathrm{C} & -1 & -4.54338000 & -3.11995500 & -3.00310800 \\ \mathrm{C} & -1 & 4.54358200 & -3.00260900 & 3.12053400 \\ \mathrm{C} & -1 & -4.53824000 & -2.98754400 & 3.13700200 \\ \mathrm{C} & -1 & 4.53842900 & -3.13763200 & -2.98699300 \\ \mathrm{Zr} & -1 & -2.51931700 & -0.00007700 & -0.00007400 \\ \mathrm{Zr} & -1 & 2.51974100 & -0.00010600 & 0.00012700 \\ \mathrm{Zr} & -1 & 0.00049300 & 1.68418800 & 1.66051400 \\ \mathrm{Zr} & -1 & -0.00020000 & 1.66051900 & -1.68417300 \\ \mathrm{Zr} & -1 & -0.00031000 & -1.66051000 & 1.68418800 \\ \mathrm{Zr} & -1 & 0.00058000 & -1.68415300 & -1.66039500 \\ \mathrm{O} & -1 & 3.70650100 & -1.49498200 & 1.50868500 \\ \mathrm{O} & -1 & 3.70185700 & -1.50999300 & -1.49629900 \\ \mathrm{O} & -1 & 2.21954900 & -2.67699600 & -2.72365400 \\ \mathrm{O} & -1 & 2.22431200 & -2.73048700 & 2.66648700 \\ \mathrm{O} & -1 & -0.03978600 & -4.37541800 & -1.53584600 \\ \mathrm{O} & -1 & 0.00152500 & -4.32620200 & 1.88199900 \\ \mathrm{O} & -1 & 3.70666300 & 1.49492000 & -1.50830700 \\ \mathrm{O} & -1 & 3.70177900 & 1.51000200 & 1.49663500 \\ \mathrm{O} & -1 & 2.22465900 & 2.73060900 & -2.66611700 \\ \mathrm{O} & -1 & 2.21934300 & 2.67704900 & 2.72376300\end{array}$




\begin{tabular}{|c|c|c|c|c|}
\hline $\mathrm{O}$ & -1 & 1.28272000 & -0.00817000 & -1.82116700 \\
\hline $\mathrm{O}$ & -1 & 1.28280600 & 0.00846700 & 1.82133800 \\
\hline $\mathrm{O}$ & -1 & -1.28165800 & -0.00825100 & -1.82555100 \\
\hline $\mathrm{O}$ & -1 & -3.70625000 & -1.50846800 & -1.49511600 \\
\hline $\mathrm{O}$ & -1 & -3.70161000 & -1.49640900 & 1.50980300 \\
\hline $\mathrm{O}$ & -1 & -2.22407500 & -2.66632500 & -2.73057700 \\
\hline $\mathrm{O}$ & -1 & -2.21930800 & -2.72377900 & 2.67681600 \\
\hline $\mathrm{O}$ & -1 & -1.28257400 & -1.82121700 & 0.00841700 \\
\hline $\mathrm{O}$ & -1 & -3.70143600 & 1.49652200 & -1.51018200 \\
\hline $\mathrm{O}$ & -1 & -3.70629100 & 1.50852700 & 1.49479200 \\
\hline $\mathrm{O}$ & -1 & -2.21897800 & 2.72361300 & -2.67726200 \\
\hline $\mathrm{O}$ & -1 & -2.22427600 & 2.66630300 & 2.73050300 \\
\hline $\mathrm{O}$ & -1 & -1.28255100 & 1.82125200 & -0.00851700 \\
\hline $\mathrm{O}$ & -1 & 0.00185700 & 4.32617800 & -1.88207000 \\
\hline $\mathrm{O}$ & -1 & -0.04002100 & 4.37542500 & 80400 \\
\hline $\mathrm{O}$ & -1 & 1.28192100 & 1.82526700 & -0.00 \\
\hline $\mathrm{O}$ & -1 & 1.28193300 & -1.82528400 & 9700 \\
\hline $\mathrm{O}$ & -1 & -1.28168000 & 0.00855200 & 1.82517400 \\
\hline $\mathrm{O}$ & -1 & 0.03972000 & -1.53576800 & 4.37545500 \\
\hline $\mathrm{O}$ & -1 & -0.00148900 & 1.88202900 & 4.32620500 \\
\hline $\mathrm{H}$ & -1 & -0.86223500 & 4.45865400 & 1.03117800 \\
\hline $\mathrm{H}$ & -1 & 0.64247100 & 4.47849700 & 0.85650300 \\
\hline $\mathrm{H}$ & -1 & -0.75619400 & 4.18595100 & -2.47584900 \\
\hline $\mathrm{H}$ & -1 & 0.78390600 & 4.18582300 & 32300 \\
\hline $\mathrm{H}$ & -1 & -0.78299900 & 2.44501100 & 32400 \\
\hline $\mathrm{H}$ & -1 & 0.75713800 & 2.47507600 & 4.18595300 \\
\hline $\mathrm{H}$ & -1 & -0.64286300 & -0.85654500 & 4.47843500 \\
\hline $\mathrm{H}$ & -1 & 0.86186300 & -1.03106300 & 4.45888900 \\
\hline $\mathrm{H}$ & -1 & 0.78265500 & -4.18581600 & 2.44549900 \\
\hline $\mathrm{H}$ & -1 & -0.75750700 & -4.18592800 & 2.47452900 \\
\hline $\mathrm{H}$ & -1 & -0.86198000 & -4.45872000 & -1.03115300 \\
\hline $\mathrm{H}$ & -1 & 0.64273200 & -4.47847800 & -0.85652100 \\
\hline $\mathrm{C}$ & -1 & 3.39712000 & 2.37268100 & -2.36999100 \\
\hline $\mathrm{C}$ & -1 & 3.39190500 & -2.37968000 & -2.36599500 \\
\hline $\mathrm{C}$ & -1 & 3.39174400 & 2.37966400 & 2.36631100 \\
\hline $\mathrm{C}$ & -1 & 3.39680700 & -2.37252100 & 2.37055500 \\
\hline $\mathrm{C}$ & -1 & -3.39137100 & 2.36604300 & -2.37999900 \\
\hline $\mathrm{C}$ & -1 & -3.39657600 & -2.37025700 & -2.37274000 \\
\hline $\mathrm{C}$ & -1 & -3.39672900 & 2.37032100 & 2.37243700 \\
\hline $\mathrm{C}$ & -1 & -3.39166900 & -2.36623000 & 2.37938100 \\
\hline $\mathrm{H}$ & -1 & 4.92838900 & -2.27735700 & 3.85211000 \\
\hline $\mathrm{H}$ & -1 & 4.24035100 & -3.90460700 & 3.66394900 \\
\hline $\mathrm{H}$ & -1 & 5.37472000 & -3.24120600 & 2.44517600 \\
\hline $\mathrm{H}$ & -1 & -4.23561900 & -3.88471200 & 3.68869800 \\
\hline $\mathrm{H}$ & -1 & -4.92093600 & -2.25472200 & 3.86211800 \\
\hline $\mathrm{H}$ & -1 & -5.37072500 & -3.23138700 & 2.4651880 \\
\hline
\end{tabular}




$\begin{array}{lrrrr}\mathrm{H} & -1 & -4.23516900 & 3.88409800 & -3.68990200 \\ \mathrm{H} & -1 & -4.91998600 & 2.25391300 & -3.86334700 \\ \mathrm{H} & -1 & -5.37063300 & 3.23069000 & -2.46676500 \\ \mathrm{H} & -1 & 4.92920500 & 2.27808100 & -3.85106900 \\ \mathrm{H} & -1 & 4.24084000 & 3.90518400 & -3.66277900 \\ \mathrm{H} & -1 & 5.37490800 & 3.24169100 & -2.44377500 \\ \mathrm{H} & -1 & -4.24011500 & -3.66322800 & -3.90518100 \\ \mathrm{H} & -1 & -4.92836700 & -3.85162800 & -2.27805100 \\ \mathrm{H} & -1 & -5.37439900 & -2.44443600 & -3.24166500 \\ \mathrm{H} & -1 & -4.24045500 & 3.66363200 & 3.90450300 \\ \mathrm{H} & -1 & -4.92860400 & 3.85158200 & 2.27726700 \\ \mathrm{H} & -1 & -5.37460700 & 2.44460700 & 3.24119900 \\ \mathrm{H} & -1 & 4.92095900 & 3.86245100 & 2.25465300 \\ \mathrm{H} & -1 & 4.23548000 & 3.68932900 & 3.88461100 \\ \mathrm{H} & -1 & 5.37070700 & 2.46574100 & 3.23165100 \\ \mathrm{H} & -1 & 4.92060200 & -3.86295500 & -2.25410000 \\ \mathrm{H} & -1 & 4.23590900 & -3.68916200 & -3.88429800 \\ \mathrm{H} & -1 & 5.37124400 & -2.46610800 & -3.23050100 \\ \mathrm{C} & -1 & -1.08136000 & 0.18665900 & -5.88805700 \\ \mathrm{C} & -1 & 1.26703900 & 0.13207700 & -5.96924300 \\ \mathrm{C} & -1 & 0.88253400 & -0.55642700 & -4.66393700 \\ \mathrm{C} & 0 & -0.61068300 & -0.80901400 & -4.82775500 \\ \mathrm{H} & 0 & -2.07393500 & 0.60545500 & -5.66299700 \\ \mathrm{H} & 0 & -1.17974500 & -0.30741300 & -6.86693800 \\ \mathrm{H} & 0 & 1.13483200 & -0.51854900 & -6.84832900 \\ \mathrm{H} & 0 & 2.30776200 & 0.48867200 & -5.97414200 \\ \mathrm{H} & 0 & 1.47086900 & -1.46950100 & -4.48747900 \\ \mathrm{H} & 0 & 1.14014900 & 0.13486200 & -3.86009600 \\ \mathrm{H} & 0 & -0.80467400 & -1.83397200 & -5.17826300 \\ \mathrm{H} & 0 & -1.20671300 & -0.68637000 & -3.91319000 \\ \mathrm{~S} & 0 & 0.11597200 & 1.51240900 & -6.07517700\end{array}$

